\title{
Ghost of vector fields in compact stars
}

\author{
Hector O. Silva $\odot,{ }^{1, *}$ Andrew Coates $\odot,{ }^{2, \dagger}$ Fethi M. Ramazanoğlu $\odot^{2, \ddagger}$ and Thomas P. Sotiriou $\odot^{3, \S}$ \\ ${ }^{1}$ Max Planck Institute for Gravitational Physics (Albert Einstein Institute), \\ Am Mühlenberg 1, 14476 Potsdam, Germany \\ ${ }^{2}$ Department of Physics, Koç University, Rumelifeneri Yolu, 34450 Sariyer, Istanbul, Turkey \\ ${ }^{3}$ School of Mathematical Sciences \& School of Physics and Astronomy, University of Nottingham, \\ University Park, Nottingham NG7 2RD, United Kingdom
}

(Received 18 October 2021; accepted 10 December 2021; published 20 January 2022)

\begin{abstract}
Spontaneous scalarization is a mechanism that allows a scalar field to go undetected in weak gravity environments and yet develop a nontrivial configuration in strongly gravitating systems. At the perturbative level it manifests as a tachyonic instability around spacetimes that solve Einstein's equations. The endpoint of this instability is a nontrivial scalar field configuration that can significantly modify a compact object's structure and can produce observational signatures of the scalar field's presence. Does such a mechanism exists for vector fields? Here we revisit the model that constitutes the most straightforward generalization of the original scalarization model to a vector field and perform a perturbative analysis. We show that a ghost appears as soon as the square of the naive effective mass squared becomes negative anywhere. This result poses a serious obstacle in generalizing spontaneous scalarization to vector fields.
\end{abstract}

DOI: 10.1103/PhysRevD.105.024046

\section{INTRODUCTION}

The first gravitational wave (GW) signal from a compact binary coalescence detected by the LIGO-Virgo collaboration [1] in 2015 opened a new vista into the nonlinear and highly dynamical regime of gravity. Moreover, and perhaps more excitingly, GWs now allow us to probe (or constrain) new physics beyond GR and the Standard Model [2-7]. This had so far been limited to astronomical probes either in the weak gravitational field and slow velocity in our Solar System or in the strong gravitational field, but small velocity and large separation regime of binary pulsars $[8,9]$.

In this context, a particularly appealing new physics scenario is one where new fundamental fields lie "dormant" in weak-gravity environments and yet manage to have significant effects in strongly-gravitating bodies and systems. The prototypical theory that achieves this was first introduced by Damour and Esposito-Farèse $[10,11]$ and involves a massless scalar field $\varphi$. The theory is described by the action

\footnotetext{
*hector.silva@aei.mpg.de

acoates@ku.edu.tr

framazanoglu@ku.edu.tr

${ }^{\S}$ Thomas.Sotiriou@nottingham.ac.uk
}

Published by the American Physical Society under the terms of the Creative Commons Attribution 4.0 International license. Further distribution of this work must maintain attribution to the author(s) and the published article's title, journal citation, and DOI. Open access publication funded by the Max Planck Society.

$$
\begin{aligned}
S_{\mathrm{DEF}}= & \frac{1}{16 \pi} \int \mathrm{d}^{4} x \sqrt{-g}\left(R-2 \nabla_{\mu} \varphi \nabla^{\mu} \varphi\right) \\
& +S_{\mathrm{m}}\left[\Psi_{\mathrm{m}} ; \Omega_{\mathrm{DEF}}^{2}(\varphi) g_{\mu \nu}\right]
\end{aligned}
$$

where $g$ is the metric determinant, $R$ is the Ricci scalar, $S_{\mathrm{m}}$ is the action of matter fields $\Psi_{\mathrm{m}}$, which couple to $\Omega_{\mathrm{DEF}}^{2}(\varphi) g_{\mu \nu}$, with $\Omega_{\mathrm{DEF}}=\exp \left(\beta \varphi^{2} / 2\right) ; \beta$ being a dimensionless constant.

The scalar field satisfies the field equation

$$
\square \varphi=-4 \pi \beta \Omega_{\mathrm{DEF}}^{4} \tilde{T} \varphi,
$$

where $\tilde{T}$ is the trace of the matter field's energy-momentum tensor. Equation (2) clearly admits a vanishing scalar field as a solution. However this is not the only solution for a given matter configuration. Linearized scalar field perturbations $\delta \varphi$ on the background of a neutron star can be shown to obey a wave equation

$$
\left(\square-\mu_{\mathrm{eff}}^{2}\right) \delta \varphi=0, \quad \mu_{\mathrm{eff}}^{2}=-4 \pi \beta \Omega_{\mathrm{DEF}}^{4} \tilde{T},
$$

where $\mu_{\text {eff }}^{2}$ is a position dependent effective mass squared. For a neutron star described by a perfect fluid, $\tilde{T}=3 \tilde{p}-\tilde{\varepsilon}$ (where $\tilde{p}$ is the pressure and $\tilde{\varepsilon}$ the total energy density). Typically $\tilde{T}<0$ and thus these perturbations can become tachyonic when $\beta<0[12,13]$, with only a weak dependence on the equation of state [14-16]. Numerical simulations show that this linear instability is ultimately nonlinearly quenched and thus the star becomes 
spontaneously scalarized. Due to Eq. (2), these scalarized stars coexist with the GR solutions [defined as stars with $\varphi=0$ ] and, importantly, are energetically favored: thus they can form dynamically from stellar collapse [14,17-20] or in neutron star binaries [21-26].

The Damour-Esposito-Farèse scalarization model cannot lead to black holes scalarization unless the latter is induced by surrounding matter [27,28]. However, more general models which fashion couplings with the GaussBonnet invariant, have been shown to lead to black hole scalarization, controlled by the mass $[29,30]$ or by the spin of the black hole [31-33] and can take place in stellar collapse [34]. Black hole scalarization can also have potentially observable effects in binary black hole binaries 37-35]] and be induced by other curvature scalars, such as the Pontryagin invariant $[38,39]$. The instability leading to scalarization can also be understood from a quantum field theory perspective, see, e.g., Refs. [40-42].

A different type of generalization of the DamourEsposito-Farèse mechanism that has been explored is to extend it to vector fields [43]. Inspired by [10,11], Ref. [43] studied the action

$$
\begin{aligned}
S_{\mathrm{v}}= & \frac{1}{16 \pi} \int \mathrm{d}^{4} x \sqrt{-g}\left(R-F_{\mu \nu} F^{\mu \nu}-2 \mu_{\mathrm{v}}^{2} A_{\mu} A^{\mu}\right) \\
& +S_{\mathrm{m}}\left[\Psi_{\mathrm{m}} ; \Omega_{\mathrm{v}}^{2}\left(A_{\mu}\right) g_{\mu \nu}\right],
\end{aligned}
$$

where $F_{\alpha \beta}=\nabla_{\alpha} A_{\beta}-\nabla_{\beta} A_{\alpha}$ is the antisymmetric Faraday tensor and $A_{\alpha}$ is a vector field with bare mass $\mu_{\mathrm{v}}{ }^{1}$ In analogy with $\Omega_{\mathrm{DEF}}$, the conformal factor is chosen as $\Omega_{\mathrm{v}}=\exp \left(\beta A_{\mu} A^{\mu} / 2\right)$, where $\beta$ is a free parameter of the theory. The field equation of $A_{\mu}$ is

$$
\nabla_{\mu} F^{\mu \alpha}=\left(\mu_{\mathrm{v}}^{2}-4 \pi \beta \Omega_{\mathrm{v}}^{4} \tilde{T}\right) A^{\alpha} .
$$

This equation promotes the bare mass $\mu_{\mathrm{v}}$ of the Proca field to what appears to be an effective mass squared $\mu_{\text {eff }}^{2}=\hat{z} \mu_{\mathrm{v}}^{2}$, where

$$
\hat{z}=1-4 \pi\left(\beta / \mu_{\mathrm{v}}^{2}\right) \Omega_{\mathrm{v}}^{4} \tilde{T},
$$

for linearized vector field perturbations. This effective mass squared can become negative in the presence of dense matter as in the theory (1). This property is not specific to the theory (4), and is shared with other vector-tensor theories with curvature coupling terms [45-48] or disformal couplings [49,50].

Based on the similarity between the field equations (2) and (5) it is natural to expect that in the theory (4), $A_{\mu}$ could also become tachyonically unstable around sufficiently compact neutron stars and a spontaneous vectorization mechanism exists. Although nonlinear vectorized neutron

\footnotetext{
${ }^{1}$ Ref. [44] is an earlier study of a similar theory that is mainly concerned with cosmology.
}

star solutions have indeed been shown to exist in [43], the perturbative manifestation of vectorization has not been explored yet. This leaves a number of open questions unanswered. In particular, a massive vector $A_{\mu}$ is known to propagate an additional longitudinal degree of freedom. What is its role in this process? Could vectorization be scalarization in disguise to some extent? More generally, can it be understood intuitively, as is the case for scalarization, as a tachyonic instability quenched by nonlinearities? Answering these questions is important from a model-building perspective, but also from a phenomenological perspective. They become even more pressing once one observes that, intuitively speaking, the aforementioned longitudinal mode gets contributions in its kinetic term from the $A^{\mu} A_{\mu}$ terms in the action. That kinetic term will therefore have a nontrivial structure, which in turn raises doubts about whether this mode is well behaved.

Motivated by these questions, here we revisit the model of Ref. [43] from a perturbative perspective and indeed uncover a ghost instability. Therefore vectorization appears to be fundamentally different from scalarization. It also strongly suggests that the time-evolution problem of a star undergoing vectorization is potentially ill-posed, casting serious doubts on the viability of this theory and other related ones. Combined with the work [51] which also found ghost (and gradient) instabilities in generalized Proca theories in compact object backgrounds, our work raises serious questions about the possibility to generalize the original mechanism of Damour and Esposito-Farèse beyond scalars since all proposed vectorization theories feature at least ghost instabilities.

The remainder of this paper explains how we arrived at these conclusions. In Secs. II and III we review the model introduced in [43], restore gauge invariance by performing the Stuckelberg trick and analyse the resulting field equations. In Sec. IV we linearize the theory's action in the background of a nonrotating, spherically symmetric star and show how ghost instability appears. In Sec. V we lift the assumption of linearized gauge field perturbations and consider the complete set of field equations. We show how ghosts, which first went unnoticed in [43], arise. In Sec. VI we summarize our main results.

We work with geometrical units $c=G=1$ and use the $(-,+,+,+)$ metric signature. Symmetrization of indices is defined as $A_{(\alpha \beta)} \equiv\left(A_{\alpha \beta}+A_{\beta \alpha}\right) / 2$ and the antisymmetrization by $A_{[\alpha \beta]} \equiv\left(A_{\alpha \beta}-A_{\beta \alpha}\right) / 2$.

\section{A MODEL FOR SPONTANEOUS VECTORIZATION WITH GAUGE SYMMETRY}

Action (4) has been constructed in analogy with (1), but a caveat of the resulting tensor-vector theory is absence of gauge invariance under $A_{\alpha} \rightarrow A_{\alpha}+\partial_{\alpha} \lambda$ ( $\lambda$ being a scalar function) due to the mass term $\mu_{\mathrm{v}}^{2} A_{\mu} A^{\mu}$. To restore gauge invariance, and at the same time more easily investigate the 
different degrees of freedom in the vector field, we can apply the Stueckelberg trick [52]. It consists of introducing a scalar field $\psi$ (the Stueckelberg field) through the substitution

$$
A_{\alpha} \rightarrow A_{\alpha}+\mu_{\mathrm{v}}^{-1} \nabla_{\alpha} \psi
$$

which results in a scalar-vector-tensor theory,

$$
\begin{aligned}
S= & \frac{1}{16 \pi} \int \mathrm{d}^{4} x \sqrt{-g}\left[R-F_{\mu \nu} F^{\mu \nu}\right. \\
& \left.-2 g^{\mu \nu}\left(\mu_{\mathrm{v}} A_{\mu}+\nabla_{\mu} \psi\right)\left(\mu_{\mathrm{v}} A_{\nu}+\nabla_{\nu} \psi\right)\right] \\
& +S_{\mathrm{m}}\left[\Psi_{\mathrm{m}} ; \Omega^{2}\left(A_{\alpha}, \nabla_{\alpha} \psi\right) g_{\mu \nu}\right],
\end{aligned}
$$

with conformal factor

$$
\ln \Omega=\frac{\beta}{2 \mu_{\mathrm{v}}^{2}} g^{\mu \nu}\left(\mu_{\mathrm{v}} A_{\mu}+\nabla_{\mu} \psi\right)\left(\mu_{\mathrm{v}} A_{\nu}+\nabla_{\nu} \psi\right) .
$$

The theory is now gauge invariant under the simultaneous transformations:

$$
A_{\alpha} \rightarrow A_{\alpha}+\nabla_{\alpha} \lambda, \quad \psi \rightarrow \psi-\mu_{\mathrm{v}} \lambda
$$

We see that $\psi$ can be set to zero by a suitable choice of $\lambda$ and thus the action (4) is a gauge-fixed version of action (8).

Indeed, for $\beta=0$ the conformal factor $\Omega$ becomes unity and we recover the Stueckelberg theory minimally coupled to gravity (see, e.g., [53]). If we fix a gauge where $\psi=0$ (we call this the "Proca gauge"), we obtain the nonminimally coupled Einstein-Proca theory of Ref. [43]. If we instead take $\mu_{\mathrm{v}} \rightarrow 0$ we obtain the Einstein-Maxwell theory with the addition of a scalar field. In Proca theory the $\mu_{\mathrm{v}} \rightarrow 0$ limit has an apparent discontinuity of the longitudinal polarization mode of $A_{\alpha}$. In the "Stueckelberged" version of the same theory, the $\mu_{\mathrm{v}} \rightarrow 0$ limit is manifestly continuous and corresponds to the decoupling between $\psi$ and $A_{\alpha}$ (the latter associated with the usual Maxwell theory). Note that, when $\beta \neq 0$, maintaining regularity of $\Omega$ requires that $\beta$ approaches zero at least as fast as $\mu_{\mathrm{v}}^{2}$ when taking the limit $\mu_{\mathrm{v}} \rightarrow 0$.

The action (8) is written in the Einstein frame (thus we call $g_{\alpha \beta}$ the Einstein frame metric). We will refer to $\tilde{g}_{\alpha \beta}=$ $\Omega^{2} g_{\alpha \beta}$ as the Jordan frame metric. We will use tildes to denote objects in the Jordan frame, some of which, as $\tilde{T}$, already appeared in the Introduction.

\section{THE FIELD EQUATIONS}

The field equations of the theory can be obtained by varying the action (8) with respect to $\psi, A_{\alpha}$ and $g^{\alpha \beta}$ :

$$
\square \psi=-\mu_{\mathrm{v}} \nabla_{\mu} A^{\mu}+\left(4 \pi / \mu_{\mathrm{v}}\right) \nabla_{\mu}\left(\alpha_{\mathrm{A}}^{\mu} \Omega^{4} \tilde{T}\right),
$$

$$
\begin{gathered}
\nabla_{\mu} F^{\mu \alpha}=\mu_{\mathrm{v}} g^{\mu \alpha}\left(\mu_{\mathrm{v}} A_{\mu}+\partial_{\mu} \psi\right)-4 \pi \alpha_{\mathrm{A}}^{\alpha} \Omega^{4} \tilde{T} \\
G_{\alpha \beta}=8 \pi\left(T_{\alpha \beta}^{\mathrm{e}}+T_{\alpha \beta}^{\mathrm{s}}+T_{\alpha \beta}\right)
\end{gathered}
$$

where,

$$
\alpha_{\mathrm{A}}^{\mu} \equiv \frac{\partial \ln \Omega}{\partial A_{\mu}}=\mu_{\mathrm{v}} \frac{\partial \ln \Omega}{\partial\left(\nabla_{\mu} \psi\right)}=\frac{\beta}{\mu_{\mathrm{v}}}\left(\mu_{\mathrm{v}} A^{\mu}+\nabla^{\mu} \psi\right),
$$

and we defined the individual energy-momentum contributions from "pure electromagnetic" theory $T_{\mu \nu}^{\mathrm{e}}$ and from the "Stueckelberg contribution" to the action $T_{\mu \nu}^{\mathrm{s}}$,

$$
\begin{aligned}
T_{\alpha \beta}^{\mathrm{e}}= & \frac{1}{4 \pi}\left(F_{\mu \alpha} F_{\nu \beta} g^{\mu \nu}-\frac{1}{4} g_{\alpha \beta} F^{\mu \nu} F_{\mu \nu}\right), \\
T_{\alpha \beta}^{\mathrm{s}}= & \frac{1}{4 \pi}\left[\left(\mu_{\mathrm{v}} A_{\alpha}+\nabla_{\alpha} \psi\right)\left(\mu_{\mathrm{v}} A_{\beta}+\nabla_{\beta} \psi\right)\right. \\
& \left.-\frac{1}{2} g_{\alpha \beta}\left(\mu_{\mathrm{v}} A^{\mu}+\nabla^{\mu} \psi\right)\left(\mu_{\mathrm{v}} A_{\mu}+\nabla_{\mu} \psi\right)\right] .
\end{aligned}
$$

The Jordan frame energy-momentum tensor of matter fields and its trace are defined as

$$
\tilde{T}_{\alpha \beta} \equiv-\frac{2}{\sqrt{-g}} \frac{\delta S_{\mathrm{m}}}{\delta \tilde{g}^{\alpha \beta}}, \quad \text { and } \quad \tilde{T} \equiv \tilde{g}^{\mu \nu} \tilde{T}_{\mu \nu}
$$

We also have by construction:

$$
\nabla_{\alpha} F_{\beta \gamma}+\nabla_{\gamma} F_{\alpha \beta}+\nabla_{\beta} F_{\gamma \alpha}=0
$$

Going back to Eq. (12) and due to $\nabla_{\mu} \nabla_{\nu} F^{\mu \nu}=0$, it is convenient to define a current $j^{\alpha}$ as:

$$
j^{\alpha}=\mu_{\mathrm{v}} g^{\mu \alpha}\left(\mu_{\mathrm{v}} A_{\mu}+\nabla_{\mu} \psi\right)-4 \pi \alpha_{\mathrm{A}}^{\alpha} \Omega^{4} \tilde{T},
$$

which is conserved

$$
\nabla_{\mu} j^{\mu}=0
$$

In terms of $j^{\alpha}$ we have:

$$
\mu_{\mathrm{v}}^{2} \nabla_{\mu} A^{\mu}=-\nabla_{\mu}\left(\mu_{\mathrm{v}} \nabla^{\mu} \psi-4 \pi \alpha_{\mathrm{A}}^{\mu} \Omega^{4} \tilde{T}\right) .
$$

In the absence of matter $(\tilde{T}=0)$ and in the Proca gauge ( $\psi=0$ ), Eq. (21) becomes the Lorenz constraint on $A_{\alpha}$ of Proca theory. Thus, the field equation for $\psi$ [cf. Eq. (11)] and the Lorenz constraint on $A_{\mu}$ are tightly connected.

We can see the first sign of the ghost by introducing a third metric,

$$
\bar{g}_{\alpha \beta}=\hat{z}^{-1} g_{\alpha \beta},
$$

in terms of which the scalar field equation becomes 
$\bar{\square} \psi=-\bar{g}^{\mu \nu}\left[\mu_{\mathrm{v}} \bar{\nabla}_{\mu} A_{\nu}+\frac{1}{2}\left(\bar{\nabla}_{\mu} \log \hat{z}\right)\left(\bar{\nabla}_{\nu} \psi+\mu_{\mathrm{v}} A_{\nu}\right)\right]$.

This third metric can, in principle, have a signature change in some parts of the spacetime due to the $\hat{z}^{-1}$ term. If this happens, the field will be a ghost in at least some region compared to any field which is coupled to a fixed signature metric. Another potential problem is the fact that this metric changes sign by diverging, rather than crossing zero, in a similar vein discussed in $[54,55]$. It is unclear whether there is a rectification for such a problem, or, worse, whether the theory can evolve from a state where this metric has a fixed signature to another where the signature changes.

It is also instructive to consider the limit $\mu_{\mathrm{v}}=0$, with $\beta \rightarrow 0$ as fast as $\mu_{\mathrm{v}}^{2}$. In this limit, the Stueckelberg field $\psi$ is no longer affected by gauge transformations, so $A_{\mu}$ becomes a gauge field. Then $A_{\mu}$ smoothly decouples from $\psi$ and the matter fields. However, there is still coupling to gravity and $\psi$ continues to be coupled to matter. In particular, Eq. (23) becomes,

$$
\bar{\square} \psi=-\frac{1}{2} \bar{g}^{\mu \nu}\left(\bar{\nabla}_{\mu} \log \hat{z}\right)\left(\bar{\nabla}_{\nu} \psi\right),
$$

note that $\hat{z}$ does not depend on $A_{\alpha}$ here. So, $\psi$ will become a ghost when the $\bar{g}_{\mu \nu}$ metric changes signature and, as it is coupled to gravity and matter, its ghostly nature is physical. This same procedure is used in the Stueckelberg picture of Proca theory to show that $\psi$ and $A_{\mu}$ decouple and hence there is no discontinuity as $\mu_{\mathrm{v}}=0$ (i.e., no degree of freedom disappears). In this setting, one has $\beta=0$, flat spacetime, and no matter.

One may object that $\psi$ can be completely removed by a gauge choice such as the Proca gauge $\psi=0$, and thus the ghost can be exorcised. For this reason we will use the rest of the paper to assuage any doubts. We will begin by examining the quadratic Lagrangian for scalar-vector perturbations around a neutron star GR solution. Doing so we will find there exists a gauge invariant scalar field that suffers the same problems.

\section{PERTURBATIVE ANALYSIS}

\section{A. Background spacetime and overview of the calculation}

In this section we explore the test-field limit of our theory, where we study the dynamics of $\psi$ and $A_{\alpha}$ in a background corresponding to a stellar solution of Einstein's field equations, i.e., a solution of the Tolman-Oppenheimer-Volkoff (TOV) equations [56,57] whose line element we write as

$\mathrm{d} s^{2}=-e^{\nu} \mathrm{d} t^{2}+\frac{r}{r-2 \mu} \mathrm{d} r^{2}+r^{2}\left(\mathrm{~d} \theta^{2}+\sin ^{2} \theta \mathrm{d} \phi\right)$,

where $\nu$ (lapse) and $\mu$ (mass function) are functions of the radial coordinate $r$ only.
In Sec. IV B we will linearize the field equations for small field perturbation $\delta \psi$ and $\delta A_{\mu}$ at the level of the field equations (11)-(12), and show how the ghost arises in this background. Then, in Sec. IV C, we reach the same conclusion by directly perturbing the Lagrangian, by expanding it to second order in the fields on the same background.

\section{B. Linearized field equations}

We are interested in studying the dynamics of $\delta A_{\alpha}$ and $\delta \psi$ propagating on the background line element (25). To proceed we decompose $\delta \psi$ and $\delta A_{\alpha}$ in scalar and vector harmonics respectively. This is the convenient basis to expand scalar and vector fields on the unit two-sphere and, thus, in problems with spherical symmetry. We follow closely the presentation by Rosa and Dolan [58], but with a slightly different normalization. More specifically, we write $\delta \psi$ as

$$
\delta \psi=\frac{1}{r} \sum_{\ell m} \sigma_{\ell m}(t, r) Y_{\ell m}(\theta, \phi),
$$

where $Y_{\ell m}=Y_{\ell m}(\theta, \phi)$ are the spherical harmonics with $\ell=0,1,2 \ldots$, and $|m| \leq \ell$. For the vector perturbations, we decompose $\delta A_{\alpha}$ as

$$
\delta A_{\alpha}=\frac{1}{r} \sum_{i=1}^{4} \sum_{\ell m} c_{i} u_{(i)}^{\ell m}(t, r) Z_{\alpha}^{(i) \ell m}(\theta, \phi)
$$

where $c_{1}=c_{2}=1, c_{3}=c_{4}=1 / \sqrt{\ell(\ell+1)}$, and $Z_{\alpha}^{(i) \ell m}$ are the vector harmonics given by

$Z_{\alpha}^{(1) \ell m}=[1,0,0,0] Y_{\ell m}$,

$Z_{\alpha}^{(2) \ell m}=[0,1,0,0] Y_{\ell m}$,

$Z_{\alpha}^{(3) \ell m}=\frac{r}{\sqrt{\ell(\ell+1)}}\left[0,0, \partial_{\theta}, \partial_{\phi}\right] Y_{\ell m}$,

$Z_{\alpha}^{(4) \ell m}=\frac{r}{\sqrt{\ell(\ell+1)}}\left[0,0, \csc \theta \partial_{\phi},-\sin \theta \partial_{\theta}\right] Y_{\ell m}$.

These functions are orthonormal when integrated on the unit two-sphere, according to the inner product,

$$
\int\left(Z_{\mu}^{(i) \ell m}\right)^{*} \eta^{\mu \nu} Z_{\nu}^{\left(i^{\prime}\right) \ell^{\prime} m^{\prime}} \sin \theta \mathrm{d} \theta \mathrm{d} \phi=\delta_{i i^{\prime}} \delta_{\ell \ell^{\prime}} \delta_{m m^{\prime}}
$$

where $\eta^{\alpha \beta} \equiv \operatorname{diag}\left[1,1,\left(1 / r^{2}\right), 1 /\left(r^{2} \sin ^{2} \theta\right)\right]$.

Under parity inversion $x \rightarrow-x^{\prime}$ (or equivalently, in spherical coordinates, $\theta \rightarrow \pi-\theta$ and $\phi \rightarrow \phi+\pi)$, the first three harmonics $(i=1,2,3)$ pick a factor of $(-1)^{\ell}$, while the fourth $(i=4)$ picks a factor of $(-1)^{\ell+1}$. We follow the literature convention and call the former "even parity" 
modes and the latter "odd parity" modes. The scalar perturbation $\delta \psi$ is of even parity.

At this point it will be useful to follow a similar procedure to [51]. We expand the Stueckelberged action (8) around a GR solution to second order in the test field approximation and find,

$$
\begin{aligned}
S_{2}[\delta A, \delta \psi]= & \frac{1}{4 \pi} \int \mathrm{d}^{4} x \sqrt{-g}\left[2\left(\nabla^{\nu} \delta A^{\mu}\right)\left(\nabla_{[\mu} \delta A_{\nu]}\right)\right. \\
& \left.-z\left(\mu_{\mathrm{v}} \delta A_{\mu}+\nabla_{\mu} \delta \psi\right)\left(\mu_{\mathrm{v}} \delta A^{\mu}+\nabla^{\mu} \delta \psi\right)\right],
\end{aligned}
$$

where,

$$
z=1-4 \pi\left(\beta / \mu_{\mathrm{v}}^{2}\right) \tilde{T},
$$

which is unity outside the star, where $\tilde{T}=0$. Note that we could have arrived at an action of this form by using the Stueckelberg trick in the Proca Lagrangian with a "dressed mass" $z \mu_{\mathrm{v}}^{2}$. Therefore, the results of this section apply to any theory whose quadratic Lagrangian can be put in this form, i.e., where one would naively expect just a screened Proca field prone to develop a tachyonic instability. Substituting the decompositions of $\delta A_{\alpha}$ and $\psi$ in harmonics, results in a Lagrangian, with even and odd-parity sector decoupled from one another. We look at each sector next.

\section{Monopolar even-parity quadratic Lagrangian}

We first focus on the monopole perturbations $(\ell=m=0$ ), which have the lowest instability threshold and belong to the even-parity sector. Since $Y_{00}=$ constant, only the $i=1,2$ vector harmonics are defined [58]. This means that we would need to work with three variables $\sigma_{00}$, $u_{00}^{(1)}$, and $u_{00}^{(2)}$,

$$
\begin{aligned}
\delta A_{\alpha} & =\frac{1}{2 \sqrt{\pi}}\left[u_{1}(t, r), u_{2}(t, r), 0,0\right], \\
\delta \psi & =\frac{1}{2 \sqrt{\pi} r} \sigma(t, r),
\end{aligned}
$$

where, to shorten the notation, we use $\sigma=\sigma_{00}, u_{1}=u_{00}^{(1)}$, and $u_{2}=u_{00}^{(2)}$ hereafter.

Inserting Eqs. (35) in the action (33) and integrating over the angular coordinates leaves us with,

$$
\begin{aligned}
S_{2}^{(\mathrm{e})}= & \int \mathrm{d} t \mathrm{~d} r \frac{e^{-\frac{\nu}{2}} \sqrt{r-2 \mu}}{4 \pi r^{5 / 2}}\left\{\frac { z } { 2 \mu _ { \mathrm { v } } ^ { 2 } } \left[\frac{r^{3}\left(\mu_{\mathrm{v}} r u_{1}+\dot{\sigma}\right)^{2}}{r-2 \mu}\right.\right. \\
& \left.\left.-e^{\nu}\left(\sigma-r\left(\mu_{\mathrm{v}} r u_{1}+\sigma^{\prime}\right)\right)^{2}\right]+\frac{r^{4}}{2}\left[u_{1}^{\prime}-\dot{u}_{2}\right]^{2}\right\},
\end{aligned}
$$

where we defined $(\cdot)^{\prime}=\partial_{r}(\cdot)$ and $(\cdot)=\partial_{t}(\cdot)$. It can be readily verified that under the gauge transformation (10) with $\lambda=l /(2 \sqrt{\pi} r)$ that, $\sigma \rightarrow \sigma-\mu_{\mathrm{v}} l, \quad\left\{u_{1}, u_{2}\right\} \rightarrow\left\{u_{1}, u_{2}\right\}+\left\{\dot{l} / r,(l / r)^{\prime}\right\}$,

and that the action (36) is invariant under this transformation. In fact, it can be verified that, the combination,

$$
\Phi=\dot{u}_{2}-u_{1}^{\prime}
$$

is itself gauge invariant (proportional to the $\ell=0$ component of the electric field). If we introduce the auxiliary field $\phi$ such that, on shell, $\phi=r^{2} \Phi$, we can rewrite Eq. (36) as,

$$
\begin{aligned}
S_{2}^{(\mathrm{e})}= & \int \mathrm{d} t \mathrm{~d} r \frac{e^{-\frac{\nu}{2}} \sqrt{r-2 \mu}}{4 \pi r^{5 / 2}}\left\{\frac{z}{2 \mu_{\mathrm{v}}^{2}}\right. \\
& \times\left[-e^{\nu}\left(\sigma-r\left(\mu_{\mathrm{v}} r u_{2}+\sigma^{\prime}\right)\right)^{2}+\frac{r^{3}\left(\mu_{\mathrm{v}} r u_{1}+\dot{\sigma}\right)^{2}}{r-2 \mu}\right] \\
& \left.+\frac{1}{2} \phi\left(2 r^{2} \Phi-\phi\right)\right\} .
\end{aligned}
$$

In this formulation, $\phi, u_{1}$, and $u_{2}$ are all nondynamical: their equations of motion can be solved algebraically in the form, e.g.,

$$
u_{1}=u_{1}\left[u_{2}, \partial u_{2}, \phi, \partial \phi, \sigma, \partial \sigma\right] .
$$

We can then replace this solution directly into the action, "integrating out" whichever field. Integrating out $u_{1}$ and $u_{2}$ one arrives at an action that is a functional of $\phi$ alone (all terms involving $\sigma$ cancel). This transfers all of the dynamics from $\sigma$ to $\phi$. The resulting action has the form,

$$
\begin{aligned}
S_{2}^{(\mathrm{e})}= & \int \mathrm{d} t \mathrm{~d} r \frac{e^{-\frac{\nu}{2}} \sqrt{r-2 \mu}}{4 \pi r^{5 / 2}}\left\{\frac { 1 } { 2 z } \left[e^{-\nu} \dot{\phi}^{2}-\left(1-\frac{2 \mu}{r}\right) \phi^{\prime 2}\right.\right. \\
& \left.\left.-\frac{2 C_{\times}}{z r^{2}} \phi \phi^{\prime}+\left(-z+\frac{z^{\prime} C_{1}}{z r^{2}}+\frac{C_{2}}{4 r^{3}}\right) \phi^{2}\right]\right\},
\end{aligned}
$$

where

$$
\begin{aligned}
& \begin{array}{c}
C_{\times}=r(r-2 \mu) z^{\prime} \\
+z\left[(r-2 \mu)\left(4+r \nu^{\prime}\right)-2 r\left(1-2 \mu^{\prime}\right)\right], \\
C_{1}=\nu^{\prime} r(r-2 \mu)+2 r \mu^{\prime}-2 \mu, \\
C_{2}=\frac{r^{2}\left(1-2 \mu^{\prime}\right)^{2}}{r-2 \mu}-\left\{6 r\left(1-2 \mu^{\prime}\right)\left(3+r \nu^{\prime}\right)+8 r^{2} \mu^{\prime \prime}\right. \\
\left.-(r-2 \mu)\left[17+r \nu^{\prime}\left(14+r \nu^{\prime}\right)-4 r^{2} \nu^{\prime \prime}\right]\right\} .
\end{array} \\
& \text { We see immediately that the sign of the kinetic con- } \\
& \text { tribution changes if } z \text { does (and also diverges when } z \\
& \text { crosses 0). That is, we have shown that, in this situation, } \\
& \text { there is a gauge invariant statement of the problems } \\
& \text { discussed in Sec. II, arising from Eq. (23). }
\end{aligned}
$$




\section{Odd-parity quadratic Lagrangian}

Having identified the presence of a ghost in the evenparity sector, it is natural to ask whether such ghosts also arise in the odd-parity sector, which contains a single degree of freedom $u_{4}$, with multipole $\ell \geq 1$. We find, after integration over the angular coordinates,

$$
\begin{aligned}
S_{2}^{(\mathrm{o})}= & \sum_{\ell=1}^{\infty} \int \mathrm{d} t \mathrm{~d} r \frac{e^{\frac{\nu}{2}}(1-2 \mu / r)^{-\frac{1}{2}}}{4 \pi \ell(\ell+1)}\left\{e^{-\nu}\left(\dot{u}_{4}\right)^{2}\right. \\
& \left.-\left(1-\frac{2 \mu}{r}\right)\left(u_{4}^{\prime}\right)^{2}-\left[\frac{\ell(\ell+1)}{r^{2}}+z \mu_{\mathrm{v}}^{2}\right] u_{4}^{2}\right\},
\end{aligned}
$$

where we defined $u_{4}=u_{\ell 0}^{(4)}$ and set $m=0$ due to the background's spherical symmetry.

Hence, we see that $u_{4}$ is prone to a tachyonic instability controlled by the same effective mass squared $z \mu_{\mathrm{v}}^{2}$ also responsible for inducing a ghost instability in the evenparity sector. Indeed, the term between square brackets is the effective potential for massive vector axial perturbations found in [58], Eq. (13), for $z=1$. We then conclude that the axial sector can become tachyonic unstable, but the dominant effect occurs at lower multipole: the ghost instability in the even-sector.

\section{UNVEILING THE GHOST IN THE PROCA GAUGE}

We have identified a ghost instability in the scalar sector of our theory, however no ghosts were reported in the spontaneous vectorization theory introduced in Ref. [43], or related theories investigated in Refs. [45-48]. In this section and related Appendices, we will demonstrate that these theories contain divergent terms in their field equations irrespective of whether one uses the Stueckelberg trick to restore gauge symmetry or not.

Recall that the Proca gauge $(\psi=0)$ is equivalent to the spontaneous vectorization theory of Ref. [43]. Effectively, this gauge undoes the Stueckelberg trick (7) and we only need to consider Eq. (5). Since there is no separate equation for $\psi$ in this picture and there are no divergent terms in this field equation, it is unclear where the ghost lurks. This is elucidated by considering the constraint equation.

Since $\nabla_{\mu} \nabla_{\nu} F^{\mu \nu}=0$ still holds due to the antisymmetry of $F^{\mu \nu}$ in Eq. (5), we obtain

$$
\nabla_{\mu}\left[\left(\mu_{\mathrm{v}}^{2}-4 \pi \Omega^{4} \beta \tilde{T}\right) A^{\mu}\right]=0 .
$$

This is the generalized version of the $\nabla_{\mu} A^{\mu}=0$ constraint for a minimally coupled Proca field.

The puzzling aspect of Eq. (5) is that it does not have any explicit indication of a ghost, however we now know from our discussion in Sec. III that the constraint (46) given in the form of a conserved current in Eq. (20) is also crucial to understand the time evolution. Indeed, the constraint imposes a time evolution for $A^{0}$ that will reveal the ghost. ${ }^{2}$

Let us rewrite the constraint in terms of $\hat{z}$ [cf. Eq. (6)],

$$
\nabla_{\mu}\left(\hat{z} A^{\mu}\right)=0
$$

We can convert the covariant derivatives to partial derivatives to obtain

$$
\partial_{0}\left(\sqrt{-g} \hat{z} A^{0}\right)=-\partial_{i}\left(\sqrt{-g} \hat{z} A^{i}\right)
$$

where $i$ runs over the spatial coordinates. We see that this time-evolution equation has divergent terms due to the behavior of $\hat{z}$, even if all fields other than $A^{0}$ are regular. Outside any matter distribution $\hat{z}=1$ and we require $\hat{z}<0$ in some part of spacetime if we want an astrophysical object to vectorize. Since $\hat{z}$ is continuous, it has to vanish at some point. There is no symmetry to ensure that $\sqrt{-g} \hat{z} A^{0}$ vanishes where $\hat{z}$ vanishes since $\hat{z}$ and its derivatives do not vanish at the same spacetime points in general. This means, $A^{0}$ will generically diverge even if $\sqrt{-g} \hat{z} A^{0}$ stays regular. Alternatively, we can move the $\hat{z}$ term outside the derivative on the left-hand side, which means that now the coefficient of the leading time derivative of $A^{0}$ vanishes at certain points. This means that the divergent terms we observed in the ghost instabilities of $\psi$ manifest themselves not directly in the field equation (5), but in the constraint equation (46), or equivalently, in Eq. (48).

The dynamics of $A^{0}$ implied by Eq. (48) is first order in time, thus not strictly of the same nature of the wave equation obeyed by $\psi$. Nonetheless, the change of sign in the time derivative leads to an analogous pathology. This can be understood by recasting the field equation (5) into an explicitly hyperbolic form.

Let us start by rearranging the constraint (47) as

$$
\nabla_{\mu}\left(\hat{z} A^{\mu}\right)=0 \Rightarrow \nabla_{\mu} A^{\mu}=-A^{\mu} \nabla_{\mu} \ln |\hat{z}|
$$

Next, we manipulate Eq. (5) as follows

$$
\begin{aligned}
\hat{z} \mu_{\mathrm{v}}^{2} A_{\alpha} & =\nabla_{\mu} F_{\alpha}^{\mu}, \\
& =\nabla_{\mu} \nabla^{\mu} A_{\alpha}-\nabla_{\mu} \nabla_{\alpha} A^{\mu}, \\
& =\square A_{\alpha}-\nabla_{\alpha} \nabla_{\mu} A^{\mu}-R^{\mu}{ }_{\nu \mu \alpha} A^{\nu}, \\
& =\square A_{\alpha}+\nabla_{\alpha}\left(A^{\mu} \nabla_{\mu} \ln |\hat{z}|\right)-R_{\alpha \mu} A^{\mu},
\end{aligned}
$$

\footnotetext{
${ }^{2}$ Note that $A^{0}$ is not a dynamical degree of freedom in the standard Hamiltonian sense [59]. The zeroth-component of the equation of motion (5), is not a time-evolution equation; it imposes an elliptic constraint on $A^{0}$ in terms of the other components of the vector and matter fields. However, one can indirectly calculate how $A^{0}$ evolves in time through the evolution of these other degrees of freedom, which can be obtained by the constraint.
} 
where we related the commutator of two covariant derivatives to the Riemann tensor in the third line, and used the constraint equation (49) in the fourth line. We finally obtain

$$
\square A_{\alpha}+\left(\nabla_{\mu} \ln |\hat{z}|\right) \nabla_{\alpha} A^{\mu}=\mathcal{M}_{\alpha \mu} A^{\mu},
$$

where we defined the mass-squared tensor

$$
\mathcal{M}_{\alpha \beta}=\hat{z} \mu_{\mathrm{v}}^{2} g_{\alpha \beta}+R_{\alpha \beta}-\nabla_{\alpha} \nabla_{\beta} \ln |\hat{z}| .
$$

We should be cautious about the fact that $\hat{z}$ contains $A^{\alpha}$ terms [inside the conformal factor; cf. Eq. (6)], which, strictly speaking, means that $\nabla_{\alpha} \nabla_{\beta} \ln |\hat{z}|$ also belongs to the principal part of the differential equation. However, for perturbative values of $A^{\alpha}$, such as in a fixed background calculation of Sec. IV, this dependence can be ignored to leading order and $\mathcal{M}_{\alpha \beta}$ becomes a proper mass-square tensor. Hence, Eq. (51) can be viewed as a generalized massive wave equation.

Equation (51) has a divergent mass term due to various factors of $\hat{z}^{-1}$ on its right-hand side. We have the option of moving these factors to the left-hand side, which means the principal part becomes $\hat{z} \square A_{\mu}$. This is a field equation prone to a ghost instability since $\hat{z}$ changes sign as we discussed before in Eq. (23). One can also analyze the equation of motion for each vector harmonic, which likewise leads to divergent effective mass terms.

The behavior of $\hat{z}$ is slightly modified for a vector field with no intrinsic mass, $\mu_{\mathrm{v}}=0$. In this case Eq. (6), and correspondingly Eq. (47), are modified as

$$
\hat{z}=-4 \pi \beta \Omega_{\mathrm{v}}^{4} \tilde{T}=-4 \pi \beta \Omega_{\mathrm{v}}^{4}(3 \tilde{p}-\tilde{\varepsilon}),
$$

where we assume the neutron star matter to behave as a perfect fluid with Jordan frame total energy density $\tilde{\varepsilon}$ and pressure $\tilde{p}$ as before. We see that $\hat{z}$ vanishes outside the star and is generally negative within it; thus it never crosses zero. However, there are still divergences.

The first case of the divergence in the field equations for $\mu_{\mathrm{v}}=0$ occurs at the surface of the neutron star. The relevant part of the TOV equations for a spherically symmetric star is [56,57],

$$
\frac{\mathrm{d} \tilde{p}}{\mathrm{~d} r}=-\frac{\tilde{\varepsilon} \mu}{r^{2}}\left(1+\frac{\tilde{p}}{\tilde{\varepsilon}}\right)\left(1+\frac{4 \pi \tilde{p} r^{3}}{\mu}\right)\left(1-\frac{2 \mu}{r}\right)^{-1} .
$$

In the outer layers of the star one has $\tilde{p} \ll \tilde{\varepsilon}$ and $4 \pi \tilde{p} r^{3} \ll \mu$ $[60,61]$, which allows us to approximate Eq. (54) as

$$
\frac{\mathrm{d} \tilde{p}}{\mathrm{~d} \varrho}=-\tilde{\rho} \mathrm{g}
$$

where we approximated the total energy-density as equal to the rest mass density $(\tilde{\varepsilon} \approx \tilde{\rho})$, introduced the proper radial length $\varrho$ [related to the coordinate radius $r$ as $\left.\mathrm{d} \varrho / \mathrm{d} r=(1-2 \mu / r)^{-1 / 2}\right]$, and defined the "local gravitational acceleration" $\mathrm{g}=\left(\mu / r^{2}\right)(1-2 \mu / r)^{-1 / 2}$ [60].

Focusing on the outer envelope of the star [62], we can approximate the spacetime as being Schwarzschild, i.e., $\mu \approx M$ and $\nu=\ln \left(1-2 M / r_{s}\right)$ in Eq. (25), where $M$ is the mass and $r_{s}$ the radius of the star. We can further introduce a local proper depth $z=(R-r)\left(1-2 M / r_{s}\right)^{-1 / 2}$, in terms of which we can recast Eq. (55) as,

$$
\frac{\mathrm{d} \tilde{p}}{\mathrm{~d} z}=\mathrm{g}_{s} \tilde{\rho},
$$

i.e., the equation of a plane-parallel atmosphere with a relativistic-corrected surface gravity $\mathrm{g}_{s}=\mathrm{g}\left(r_{s}\right)=\left(M / r_{s}\right)$ $\left(1-2 M / r_{s}\right)^{-1 / 2}$. In the outermost stellar layers, the main contribution to the pressure is due to a nonrelativistic degenerate electron gas, for which Eq. (56) can be solved exactly (see Ref. [60], Sec. 6.9), yielding the scaling $\tilde{\rho} \propto 3^{3 / 2}$ and, within the same assumptions, $\tilde{T} \propto-4 \pi \beta z^{3 / 2}$. This, in turn, means that $\nabla_{\mu} \ln |\hat{z}|$ and the effective mass diverge on the surface, completing our argument. The same reasoning can in principle be applied to other systems which have an interface of vacuum and matter, suggesting that any such interfaces would lead to a divergence in the vector field equations in general. These divergences at the surface of the star are not exclusive to the vector-tensor model considered here, but are known to also arise, albeit with a different origin, in Palatini $f(R)$ [63-65] and in Eddington-inspired BornInfeld [66] theories. See also [67,68].

The second case of divergence in the field equations for $\mu_{\mathrm{v}}=0$ is related to massive neutron stars. Although $\tilde{T}$ is negative in general, it can switch sign and become positive in the core of such stars for some equations of state (see e.g., [69-72]). This means that $\hat{z}$ vanishes somewhere inside the star [cf. Eq. (53)], where our previous results for the $\mu_{\mathrm{v}} \neq 0$ case directly apply.

Overall, the above discussion provides a heuristic tool to identify ghosts in spontaneous vectorization theories. If the spacetime dependent $\mu_{\text {eff }}^{2}$ vanishes in nonvacuum regions in a theory with field equation $\nabla_{\mu} F^{\mu \alpha}=\mu_{\text {eff }}^{2} A^{\alpha}$, this generically leads to divergent terms in the explicitly hyperbolic field equations. In other words, despite the appearances and the naming we used, $\mu_{\text {eff }}$ is not the effective mass of all physical degrees of freedom. A careful analysis reveals that the true effective mass diverges as in Eq. (51), which was overlooked in the original spontaneous vectorization theory of Ref. [43] and other similar theories. We work this out explicitly in Appendix A (for the Hellings-Nordtvedt vector-tensor theory $[73,74]$ studied in [45]) and in Appendix B (for the vectorGauss-Bonnet theory of $[46,48])$.

\section{CONCLUSIONS}

We revisited the tensor-vector gravity model proposed in Ref. [43] and explored the vectorization process using 
perturbation theory. This was done by working with a gauge invariant, Stueckelberg version of the theory and complemented with an analysis of the Lorenz constraint in the Proca gauge. In analogy with scalarization, one would expect to see the vector field develops a tachyonic instability, which is then quenched nonlinearly, and this process gives rise to the vectorized configurations found in previous work. Instead, we have uncovered a ghost instability. This results demonstrates quite clearly that the strong resemblance of this model of vectorization to the Damour-Esposito-Farèse model of scalarization is in fact rather misleading and a phase transition process that is physically similar to scalarization does not take place.

A potential way out may exist if one can tame the ghost instability nonlinearly, similar to the quenching of the tachyonic instability in scalarization. Indeed, "ghost-based spontaneous tensorization" has been investigated [75]. In the vectorization model studied here, ghosts appear inadvertently, and there is no explicit derivative coupling before the introduction of the Stueckelberg mechanism. Yet, if a nonlinear quenching mechanism exists, it could, in principle, suppress the ghost. Note that the $\hat{z}$ term in Eq. (47) that controls the instability approaches its GR value of $\hat{z}=1$ when $A_{\mu} A^{\mu} \rightarrow \infty$ (for $\beta<0$ ). Hence, a solution with large vector field values can lead to a case where $\hat{z}>1$ everywhere. This possibility was recently investigated for action (4) in Ref. [76], and all computed static and spherically symmetric vectorized neutron stars were shown to still carry ghost or gradient instabilities. Hence, there is no sign of a quenching of the instabilities so far.

The main issue however with the ghost instabilities we investigated is that it is not known whether their time evolution can be done. Even if a vector field growing to large values might quench the ghost, it is not clear if the very time evolution of the vector field that leads to growth can be formulated as a well-posed initial value problem due to the divergent terms such as those in Eq. (51). The resolution of this issue requires a mathematical analysis of the partial differential equations we have, which is beyond the scope of this work. We remark that these are not problems in the Proca limit of our model and in the absence of matter, in which numerical relativity simulations have been performed, e.g., in Refs. [77-79].

Spontaneous vectorization theories with restored gauge symmetry were also conceived using the Higgs mechanism rather than the Stueckelberg mechanism [80], inspired by the gravitational Higgs mechanism [81-83]. However, this theory [80] also has divergent terms in its field equations akin to Eq. (50), hence, it is susceptible to the same illposedness problems we discussed here.

We worked on the specific theory of Eq. (5), but other spontaneous vectorization models in the literature have similar field equations where $\nabla_{\mu} F^{\mu \alpha}$ directly appears as the principal part [44-49]. Hence, a constraint can be obtained the same way as we did, which leads to divergent terms using the arguments in Sec. Vor related ones, as we show in Appendices A and B.

Lastly, we stress that our results are relevant for most known extensions of spontaneous scalarization to other fields, not just the vectors, and our study can be considered as a first step to obtain a no-go theorem for extending spontaneous scalarization to other fields. For vector fields, Garcia-Saenz et al. [51] has identified the presence of ghost and gradient instabilities in the background of compact objects in a broad class of generalized Proca theories $[84,85]$. Similar concerns were also raised in the context of cosmology in Ref. [86]. Going beyond vector fields, all known formulations of nonminimally coupled spin-2 fields that could spontaneously grow are known to lead to ghost instabilities as well [75]. Likewise, $p$-form fields also have the same constraint structure we discussed in Sec. V, hence they suffer from similar divergent terms [87]. Spontaneous growth of spinor fields as it was introduced in Ref. [88] also contains divergent terms.

The only potential exception to our long list of problematic theories is a second form of spontaneous spinorization theory proposed in Ref. [89], whose equations of motion are not known to feature divergences. It remains to be seen if other well-posed theories exist. If this is the case, understanding what distinguishes these theories at a fundamental level from the problematic ones may lead to a proper no-go theorem for arbitrary generalizations of spontaneous scalarization.

\section{ACKNOWLEDGMENTS}

We thank Leonardo Gualtieri, Kirill Krasnov, Helvi Witek and Jun Zhang for discussions. T. P. S. acknowledges partial support from the STFC Consolidated Grants No. ST/ T000732/1 and No. ST/V005596/1. F. M. R. and A.C. were supported by Grant No. 117F295 of the Scientific and Technological Research Council of Turkey (TÜBİTAK). A.C. acknowledges financial support from the European Commission and TÜBITAK under the CO-FUNDED Brain Circulation Scheme 2, Project No. 120C081. F. M. R. is also supported by a Young Scientist (BAGEP) Award of Bilim Akademisi of Turkey. H. O. S. thanks the hospitality of the University of Nottingham where this work started. The authors also acknowledge networking support by the GWverse COST Action CA16104, "Black holes, gravitational waves and fundamental physics". Some of our calculations were performed with the Mathematica packages XPERT [90] and XCOBA, parts of the XACT/XTENSOR suite $[91,92]$.

\section{APPENDIX A: THE HELLINGS-NORDTVEDT THEORY}

In this Appendix we apply the approach of Sec. V to examine the field equations in the Hellings-Nordtvedt 
$[73,74]$ vector-tensor theory studied in Ref. [45] as a vectorization model.

In this theory, the vector field obeys the field equation,

$$
\nabla_{\mu} F^{\mu \alpha}-\frac{1}{2} \omega R A^{\alpha}-\frac{1}{2} \eta R_{\mu}^{\alpha} A^{\mu}=0,
$$

where $\omega$ and $\eta$ are dimensionless coupling constants.

Let us first obtain a generalized Lorenz constraint satisfied by $A^{\alpha}$ by taking a covariant derivative of Eq. (A1) and using $\nabla_{\nu} \nabla_{\mu} F^{\nu \mu}=0$,

$$
\nabla_{\mu}\left(\omega R A^{\mu}+\eta R_{\nu}^{\mu} A^{\nu}\right)=0 .
$$

We can expand this equation and replace the Ricci tensor with the Einstein tensor and the Ricci scalar. The resulting constraint equation is,

$\nabla_{\mu} A^{\mu}+A^{\mu} \nabla_{\mu} \ln |\omega R|+\frac{2 \eta}{\eta+2 \omega} \frac{1}{R} G^{\mu}{ }_{\nu} \nabla_{\mu} A^{\nu}=0$.

We can now return to Eq. (A1), write $F^{\alpha \beta}$ in terms of $A^{\alpha}$, follow the same steps that lead to Eq. (50), and find:

$$
\square A_{\alpha}-\nabla_{\alpha} \nabla_{\mu} A^{\mu}-\left[\frac{1}{2} \omega R g_{\alpha \mu}+\left(1+\frac{\eta}{2}\right) R_{\alpha \mu}\right] A^{\mu}=0 .
$$

At last, using Eq. (A3) we obtain,

$$
\begin{aligned}
& \square A_{\alpha}+\nabla_{\mu} \ln (|\omega R|) \nabla_{\alpha} A^{\mu}+\nabla_{\alpha}\left(\frac{2 \eta}{\eta+2 \omega} \frac{1}{R} G^{\mu}{ }_{\nu} \nabla_{\mu} A^{\nu}\right) \\
& -\mathcal{M}_{\alpha \mu} A^{\mu}=0,
\end{aligned}
$$

where

$\mathcal{M}_{\alpha \beta}=\frac{1}{2} \omega R g_{\alpha \beta}+\left(1+\frac{\eta}{2}\right) R_{\alpha \beta}-\nabla_{\alpha} \nabla_{\beta} \ln |\omega R|$,

which should be compared against Eq. (52). Note that in Eq. (A5) the last term in the first line is also second order, hence, it contributes to the principal part of the differential equation in addition to the wave operator. Hence, this equations is not in an explicitly hyperbolic form, and we cannot immediately identify $\mathcal{M}_{\alpha \beta}$ as a squared-mass tensor whose eigenvalues are related to the effective masses of the individual degrees of freedom. However, such identification is possible in the special case $\eta=0$ in which the problematic term vanishes and then:

$$
\mathcal{M}_{\alpha \beta}^{(\eta=0)}=(\omega / 2) R g_{\alpha \beta}+R_{\alpha \beta}-\nabla_{\alpha} \nabla_{\beta} \ln |\omega R|
$$

We see, by comparing with Eqs. (A7) and (52), that $\omega R$ plays the role of $\hat{z}$. We then conclude that a ghost arises for the same reasons discussed in Sec. V.

For the general case $\eta \neq 0$ it is more convenient to analyse the constraint (A2) which we write as,

$$
\nabla_{\alpha}\left(\Xi_{\beta}^{\alpha} A^{\beta}\right)=0
$$

where

$$
\Xi_{\beta}^{\alpha}=\eta G_{\beta}^{\alpha}+(\omega+\eta / 2) R \delta_{\beta}^{\alpha} .
$$

Let us focus on the perturbative regime where the background metric is fixed and the Einstein equations hold, i.e., $G_{\alpha \beta}=8 \pi T_{\alpha \beta}$ [45]. For a static, spherically symmetric perfect fluid star with energy density $\varepsilon$ and pressure $p$,

$$
\begin{aligned}
\Xi^{\alpha}{ }_{\beta}= & 4 \pi \eta\left[(\varepsilon-p) \delta^{\alpha}{ }_{\beta}-2(\varepsilon+p) \delta^{\alpha}{ }_{0} \delta^{0}{ }_{\beta}\right] \\
& +8 \pi \omega(\varepsilon-3 p) \delta^{\alpha}{ }_{\beta},
\end{aligned}
$$

which is diagonal. The constraint can then be written as

$$
\partial_{0}\left(\sqrt{-g} \Xi_{0}^{0} A^{0}\right)=-\sum_{k} \partial_{k}\left(\sqrt{-g} \Xi_{k}^{k} A^{k}\right),
$$

where we wrote the summation over the spatial coordinates $k$ explicitly to avoid confusion. This means the diagonal elements have the role of a generalized $\hat{z}$ in the massless case in Eq. (53). We see that $\partial_{0} A^{0}$ has a contribution in the form of

$$
\partial_{0} A^{0}=-\frac{\partial_{r}\left(\Xi_{r}^{r}\right)}{\Xi_{0}^{0}} A^{r}+\ldots
$$

The behavior of this term is given by the dependence of the energy density and the pressure on the radial coordinate at the surface of the star. We normally encounter power law dependence in stars due to the TOV equations as we mentioned in relation to Eq. (53). Hence, $\partial_{0} A^{0}$ diverges for generic configurations of $A^{\mu}$.

We conclude by noticing that the constraint equations of disformally coupled vector-tensor theories of Refs. [49,50] have a similar structure to Eq. (A8), which would lead to similar results in terms of divergences.

\section{APPENDIX B: VECTOR-GAUSS-BONNET THEORY}

In this Appendix we apply the approach of Sec. V to examine the field equations in the vector-Gauss-Bonnet theory introduced in Ref. [46], and further studied in Ref. [48]. The motivation behind these theories is to 
generalize the spontaneous scalarization of black holes $[29,30]$ to vector fields.

In this theory, the vector field obeys the field equation,

$$
\nabla_{\mu} F^{\mu \alpha}=v A^{\alpha}-f \mathcal{G} A^{\alpha},
$$

with

$$
v=\frac{1}{2} \frac{\mathrm{d} V\left(A_{\mu} A^{\mu}\right)}{\mathrm{d}\left(A_{\mu} A^{\mu}\right)}, \quad f=\frac{1}{2} \frac{\mathrm{d} F\left(A_{\mu} A^{\mu}\right)}{\mathrm{d}\left(A_{\mu} A^{\mu}\right)}
$$

where $V$ is the vector field's self-interaction potential, $F$ prescribes the coupling between the vector field and $\mathcal{G}$, the Gauss-Bonnet invariant. For small field perturbations, the potential $V$ and coupling function $F$ considered in Ref. [48] reduce to:

$$
v=\mu_{\mathrm{v}}^{2}, \quad f=\beta / 2,
$$

where $\mu_{\mathrm{v}}^{2}$ is the bare mass of $A^{\alpha}$ and $\beta$ a coupling constant. As with Eq. (5), one can identify an "effective mass squared" $\mu_{\text {eff }}^{2}=\hat{z} \mu_{\mathrm{v}}^{2}$, but where now $\hat{z}=1-\left(\beta / \mu_{\mathrm{v}}^{2}\right) \mathcal{G} / 2$.

We can now proceed in the same manner as in Sec. $\mathrm{V}$ to obtain

$$
\square A_{\alpha}+\left(\nabla_{\mu} \ln |\hat{z}|\right) \nabla_{\alpha} A^{\mu}-\mathcal{M}_{\alpha \mu} A^{\mu}=0,
$$

where

$$
\mathcal{M}_{\alpha \beta}=\mu_{\mathrm{v}}^{2} \hat{z} g_{\alpha \beta}-\nabla_{\beta} \nabla_{\alpha} \ln |\hat{z}|,
$$

[compare against Eqs. (51)-(52)] where the absence of the Ricci tensor is due to the assumption of the GR background being Ricci flat [48]. Therefore, this theory suffers from a ghost instability as the one considered in Ref. [43].

For a Schwarzschild black hole, $\mathcal{G}$ is positive everywhere. Thus, for $v=0$ the above argument cannot be repeated verbatim. However, $\mathcal{G}$, and hence the effective mass squared, changes sign in some regions outside the event horizon of black holes with dimensionless spin $\gtrsim 0.5$ [93]. Therefore, these commonly encountered astrophysical systems lead to divergent field equations in such theories.

Neutron stars also feature divergences for the case of $v=0$. On a fixed general relativistic background, the Gauss-Bonnet invariant of a static spherically symmetric perfect fluid star of energy density $\varepsilon$ and pressure $p$ is given by [30]

$$
\mathcal{G}(r)=\frac{48 \mu^{2}}{r^{6}}-128 \pi\left(2 \pi p+\frac{\mu}{r^{3}}\right) \varepsilon,
$$

which is positive definite outside the star. On the other hand, near the center of the star $r=r_{c}$, the TOV equations imply that the mass function is approximately

$$
\mu_{c}=\mu\left(r_{c}\right) \approx \frac{4}{3} \pi \varepsilon_{c} r_{c}^{3},
$$

where $\varepsilon_{c}=\varepsilon\left(r_{c}\right)$ is the central energy density. We then find that in the star's center,

$$
\mathcal{G}_{c} \approx-256 \pi^{2}\left(p_{c}+\varepsilon_{c} / 3\right) \varepsilon_{c}
$$

where $p_{c}=p\left(r_{c}\right)$ is the central pressure. The right-hand side of Eq. (B8) is negative meaning that $\mathcal{G}$, and thus $\mu_{\text {eff }}^{2}$, change sign within the star, numerically found to happen near the surface [30].
[1] B. P. Abbott et al. (LIGO Scientific, Virgo Collaborations), Observation of Gravitational Waves from a Binary Black Hole Merger, Phys. Rev. Lett. 116, 061102 (2016).

[2] E. Berti et al., Testing general relativity with present and future astrophysical observations, Classical Quantum Gravity 32, 243001 (2015).

[3] N. Yunes, K. Yagi, and F. Pretorius, Theoretical physics implications of the binary black-hole mergers GW150914 and GW151226, Phys. Rev. D 94, 084002 (2016).

[4] L. Barack et al., Black holes, gravitational waves and fundamental physics: A roadmap, Classical Quantum Gravity 36, 143001 (2019).

[5] R. Nair, S. Perkins, H. O. Silva, and N. Yunes, Fundamental Physics Implications for Higher-Curvature Theories from
Binary Black Hole Signals in the LIGO-Virgo Catalog GWTC-1, Phys. Rev. Lett. 123, 191101 (2019).

[6] R. Abbott et al. ( LIGO Scientific, Virgo Collaborations), Tests of general relativity with binary black holes from the second LIGO-Virgo gravitational-wave transient catalog, Phys. Rev. D 103, 122002 (2021).

[7] S. E. Perkins, R. Nair, H. O. Silva, and N. Yunes, Improved gravitational-wave constraints on higher-order curvature theories of gravity, Phys. Rev. D 104, 024060 (2021).

[8] C. M. Will, The confrontation between general relativity and experiment, Living Rev. Relativity 17, 4 (2014).

[9] N. Wex and M. Kramer, Gravity tests with radio pulsars, Universe 6, 156 (2020). 
[10] T. Damour and G. Esposito-Farèse, Nonperturbative Strong Field Effects in Tensor-Scalar Theories of Gravitation, Phys. Rev. Lett. 70, 2220 (1993).

[11] T. Damour and G. Esposito-Farèse, Tensor-scalar gravity and binary pulsar experiments, Phys. Rev. D 54, 1474 (1996).

[12] T. Harada, Stability analysis of spherically symmetric star in scalar-tensor theories of gravity, Prog. Theor. Phys. 98, 359 (1997).

[13] T. Chiba, T. Harada, and K.-i. Nakao, Gravitational physics in scalar-tensor theories: tests of strong field gravity, Prog. Theor. Phys. Suppl. 128, 335 (1997).

[14] J. Novak, Neutron star transition to strong-scalar-field state in tensor scalar gravity, Phys. Rev. D 58, 064019 (1998).

[15] H. O. Silva, C. F. B. Macedo, E. Berti, and L. C. B. Crispino, Slowly rotating anisotropic neutron stars in general relativity and scalar-tensor theory, Classical Quantum Gravity 32, 145008 (2015).

[16] Z. Altaha Motahar, J. L. Blázquez-Salcedo, B. Kleihaus, and J. Kunz, Scalarization of neutron stars with realistic equations of state, Phys. Rev. D 96, 064046 (2017).

[17] J. Novak and J. M. Ibáñez, Gravitational waves from the collapse and bounce of a stellar core in tensor scalar gravity, Astrophys. J. 533, 392 (2000).

[18] D. Gerosa, U. Sperhake, and C. D. Ott, Numerical simulations of stellar collapse in scalar-tensor theories of gravity, Classical Quantum Gravity 33, 135002 (2016).

[19] U. Sperhake, C. J. Moore, R. Rosca, M. Agathos, D. Gerosa, and C. D. Ott, Long-Lived Inverse Chirp Signals from Core Collapse in Massive Scalar-Tensor Gravity, Phys. Rev. Lett. 119, 201103 (2017).

[20] R. Rosca-Mead, U. Sperhake, C. J. Moore, M. Agathos, D. Gerosa, and C. D. Ott, Core collapse in massive scalartensor gravity, Phys. Rev. D 102, 044010 (2020).

[21] E. Barausse, C. Palenzuela, M. Ponce, and L. Lehner, Neutron-star mergers in scalar-tensor theories of gravity, Phys. Rev. D 87, 081506 (2013).

[22] C. Palenzuela, E. Barausse, M. Ponce, and L. Lehner, Dynamical scalarization of neutron stars in scalar-tensor gravity theories, Phys. Rev. D 89, 044024 (2014).

[23] M. Shibata, K. Taniguchi, H. Okawa, and A. Buonanno, Coalescence of binary neutron stars in a scalar-tensor theory of gravity, Phys. Rev. D 89, 084005 (2014).

[24] K. Taniguchi, M. Shibata, and A. Buonanno, Quasiequilibrium sequences of binary neutron stars undergoing dynamical scalarization, Phys. Rev. D 91, 024033 (2015).

[25] N. Sennett and A. Buonanno, Modeling dynamical scalarization with a resummed post-Newtonian expansion, Phys. Rev. D 93, 124004 (2016).

[26] N. Sennett, L. Shao, and J. Steinhoff, Effective action model of dynamically scalarizing binary neutron stars, Phys. Rev. D 96, 084019 (2017).

[27] V. Cardoso, I. P. Carucci, P. Pani, and T. P. Sotiriou, Black Holes with Surrounding Matter in Scalar-Tensor Theories, Phys. Rev. Lett. 111, 111101 (2013).

[28] V. Cardoso, I. P. Carucci, P. Pani, and T. P. Sotiriou, Matter around Kerr black holes in scalar-tensor theories: Scalarization and superradiant instability, Phys. Rev. D 88, 044056 (2013).
[29] D. D. Doneva and S. S. Yazadjiev, New Gauss-Bonnet Black Holes with Curvature-Induced Scalarization in Extended Scalar-Tensor Theories, Phys. Rev. Lett. 120, 131103 (2018).

[30] H. O. Silva, J. Sakstein, L. Gualtieri, T. P. Sotiriou, and E. Berti, Spontaneous Scalarization of Black Holes and Compact Stars from a Gauss-Bonnet Coupling, Phys. Rev. Lett. 120, 131104 (2018).

[31] A. Dima, E. Barausse, N. Franchini, and T. P. Sotiriou, SpinInduced Black Hole Spontaneous Scalarization, Phys. Rev. Lett. 125, 231101 (2020).

[32] C. A. R. Herdeiro, E. Radu, H. O. Silva, T. P. Sotiriou, and N. Yunes, Spin-Induced Scalarized Black Holes, Phys. Rev. Lett. 126, 011103 (2021).

[33] E. Berti, L. G. Collodel, B. Kleihaus, and J. Kunz, SpinInduced Black-Hole Scalarization in Einstein-scalar-GaussBonnet Theory, Phys. Rev. Lett. 126, 011104 (2021).

[34] H.-J. Kuan, D. D. Doneva, and S. S. Yazadjiev, Dynamical Formation of Scalarized Black Holes and Neutron Stars through Stellar Core Collapse, Phys. Rev. Lett. 127, 161103 (2021).

[35] H. O. Silva, H. Witek, M. Elley, and N. Yunes, Dynamical Descalarization in Binary Black Hole Mergers, Phys. Rev. Lett. 127, 031101 (2021).

[36] W. E. East and J. L. Ripley, Evolution of Einstein-scalarGauss-Bonnet gravity using a modified harmonic formulation, Phys. Rev. D 103, 044040 (2021).

[37] W. E. East and J. L. Ripley, Dynamics of Spontaneous Black Hole Scalarization and Mergers in Einstein-Scalar-GaussBonnet Gravity, Phys. Rev. Lett. 127, 101102 (2021).

[38] Y.-X. Gao, Y. Huang, and D.-J. Liu, Scalar perturbations on the background of Kerr black holes in the quadratic dynamical Chern-Simons gravity, Phys. Rev. D 99, 044020 (2019).

[39] D. D. Doneva and S. S. Yazadjiev, Spontaneously scalarized black holes in dynamical Chern-Simons gravity: Dynamics and equilibrium solutions, Phys. Rev. D 103, 083007 (2021).

[40] W. C. C. Lima, G. E. A. Matsas, and D. A. T. Vanzella, Awaking the Vacuum in Relativistic Stars, Phys. Rev. Lett. 105, 151102 (2010).

[41] R. F. P. Mendes, G. E. A. Matsas, and D. A. T. Vanzella, Quantum versus classical instability of scalar fields in curved backgrounds, Phys. Rev. D 89, 047503 (2014).

[42] P. Pani, V. Cardoso, E. Berti, J. Read, and M. Salgado, The vacuum revealed: the final state of vacuum instabilities in compact stars, Phys. Rev. D 83, 081501 (2011).

[43] F. M. Ramazanoğlu, Spontaneous growth of vector fields in gravity, Phys. Rev. D 96, 064009 (2017).

[44] J. Beltran Jimenez, A. L. Delvas Froes, and D. F. Mota, Screening vector field modifications of general relativity, Phys. Lett. B 725, 212 (2013).

[45] L. Annulli, V. Cardoso, and L. Gualtieri, Electromagnetism and hidden vector fields in modified gravity theories: spontaneous and induced vectorization, Phys. Rev. D 99, 044038 (2019).

[46] F. M. Ramazanoğlu, Spontaneous tensorization from curvature coupling and beyond, Phys. Rev. D 99, 084015 (2019). 
[47] R. Kase, M. Minamitsuji, and S. Tsujikawa, Neutron stars with a generalized Proca hair and spontaneous vectorization, Phys. Rev. D 102, 024067 (2020).

[48] S. Barton, B. Hartmann, B. Kleihaus, and J. Kunz, Spontaneously vectorized Einstein-Gauss-Bonnet black holes, Phys. Lett. B 817, 136336 (2021).

[49] F. M. Ramazanoğlu and K. I. Ünlütürk, Generalized disformal coupling leads to spontaneous tensorization, Phys. Rev. D 100, 084026 (2019).

[50] M. Minamitsuji, Spontaneous vectorization in the presence of vector field coupling to matter, Phys. Rev. D 101, 104044 (2020).

[51] S. Garcia-Saenz, A. Held, and J. Zhang, Destabilization of Black Holes and Stars by Generalized Proca Fields, Phys. Rev. Lett. 127, 131104 (2021).

[52] H. Ruegg and M. Ruiz-Altaba, The Stueckelberg field, Int. J. Mod. Phys. A 19, 3265 (2004).

[53] A. Belokogne and A. Folacci, Stueckelberg massive electromagnetism in curved spacetime: Hadamard renormalization of the stress-energy tensor and the Casimir effect, Phys. Rev. D 93, 044063 (2016).

[54] M. Minamitsuji and H. O. Silva, Relativistic stars in scalartensor theories with disformal coupling, Phys. Rev. D 93, 124041 (2016).

[55] G. Ventagli, A. Lehébel, and T. P. Sotiriou, Onset of spontaneous scalarization in generalized scalar-tensor theories, Phys. Rev. D 102, 024050 (2020).

[56] R. C. Tolman, Static solutions of Einstein's field equations for spheres of fluid, Phys. Rev. 55, 364 (1939).

[57] J. R. Oppenheimer and G. M. Volkoff, On massive neutron cores, Phys. Rev. 55, 374 (1939).

[58] J. G. Rosa and S. R. Dolan, Massive vector fields on the Schwarzschild spacetime: Quasi-normal modes and bound states, Phys. Rev. D 85, 044043 (2012).

[59] L. Heisenberg, A systematic approach to generalisations of general relativity and their cosmological implications, Phys. Rep. 796, 1 (2019).

[60] P. Haensel, A. Y. Potekhin, and D. G. Yakovlev, Neutron stars 1: Equation of State and Structure (Springer, New York, USA, 2007), Vol. 326.

[61] K. Y. Ekşi, Neutron stars: Compact objects with relativistic gravity, Turk. J. Phys. 40, 127 (2016).

[62] V. A. Urpin and D. G. Yakovlev, On the growth of temperature within neutron stars, Astrophysics 15, 429 (1979).

[63] E. Barausse, T.P. Sotiriou, and J. C. Miller, A No-go theorem for polytropic spheres in Palatini $f(R)$ gravity, Classical Quantum Gravity 25, 062001 (2008).

[64] E. Barausse, T.P. Sotiriou, and J.C. Miller, Curvature singularities, tidal forces and the viability of Palatini $f(R)$ gravity, Classical Quantum Gravity 25, 105008 (2008).

[65] E. Barausse, T. P. Sotiriou, and J. C. Miller, Polytropic spheres in Palatini $f(R)$ gravity, EAS Publ. Ser. 30, 189 (2008).

[66] P. Pani and T. P. Sotiriou, Surface Singularities in Eddington-Inspired Born-Infeld Gravity, Phys. Rev. Lett. 109, 251102 (2012).

[67] T. P. Sotiriou, The Viability of theories with matter coupled to the Ricci scalar, Phys. Lett. B 664, 225 (2008).
[68] P. Pani, T. P. Sotiriou, and D. Vernieri, Gravity with auxiliary fields, Phys. Rev. D 88, 121502 (2013).

[69] R. F. P. Mendes, Possibility of setting a new constraint to scalar-tensor theories, Phys. Rev. D 91, 064024 (2015).

[70] C. Palenzuela and S. L. Liebling, Constraining scalar-tensor theories of gravity from the most massive neutron stars, Phys. Rev. D 93, 044009 (2016).

[71] R. F. P. Mendes and N. Ortiz, Highly compact neutron stars in scalar-tensor theories of gravity: Spontaneous scalarization versus gravitational collapse, Phys. Rev. D 93, 124035 (2016).

[72] D. M. Podkowka, R. F. P. Mendes, and E. Poisson, Trace of the energy-momentum tensor and macroscopic properties of neutron stars, Phys. Rev. D 98, 064057 (2018).

[73] R. W. Hellings and K. Nordtvedt, Vector-Metric Theory of Gravity, Phys. Rev. D 7, 3593 (1973).

[74] C. M. Will, Theory and Experiment in Gravitational Physics (Cambridge University Press, Cambridge, England, 1993).

[75] F. M. Ramazanoğlu, Regularization of instabilities in gravity theories, Phys. Rev. D 97, 024008 (2018); 99, 069905(E) (2019).

[76] E.S. Demirboğa, A. Coates, and F. M. Ramazanoğlu, Instability of vectorized stars, arXiv:2112.04269.

[77] M. Zilhão, H. Witek, and V. Cardoso, Nonlinear interactions between black holes and Proca fields, Classical Quantum Gravity 32, 234003 (2015).

[78] W. E. East and F. Pretorius, Superradiant Instability and Backreaction of Massive Vector Fields around Kerr Black Holes, Phys. Rev. Lett. 119, 041101 (2017).

[79] W. E. East, Superradiant instability of massive vector fields around spinning black holes in the relativistic regime, Phys. Rev. D 96, 024004 (2017).

[80] F. M. Ramazanoğlu, Spontaneous growth of gauge fields in gravity through the Higgs mechanism, Phys. Rev. D 98, 044013 (2018).

[81] A. Coates, M. W. Horbartsch, and T. P. Sotiriou, Gravitational Higgs mechanism in neutron star interiors, Phys. Rev. D 95, 084003 (2017).

[82] N. Franchini, A. Coates, and T. P. Sotiriou, Constructing neutron stars with a gravitational Higgs mechanism, Phys. Rev. D 97, 064013 (2018).

[83] V. Krall, A. Coates, and K. D. Kokkotas, Gravitational Higgs mechanism and resulting observational effects, Phys. Rev. D 102, 124065 (2020).

[84] G. Tasinato, Cosmic acceleration from abelian symmetry breaking, J. High Energy Phys. 04 (2014) 067.

[85] L. Heisenberg, Generalization of the proca action, J. Cosmol. Astropart. Phys. 05 (2014) 015.

[86] G. Esposito-Farèse, C. Pitrou, and J.-P. Uzan, Vector theories in cosmology, Phys. Rev. D 81, 063519 (2010).

[87] F. M. Ramazanoğlu, Various paths to the spontaneous growth of $p$-form fields, Turk. J. Phys. 43, 586 (2019).

[88] F. M. Ramazanoğlu, Spontaneous growth of spinor fields in gravity, Phys. Rev. D 98, 044011 (2018); 100, 029903(E) (2019).

[89] M. Minamitsuji, Stealth spontaneous spinorization of relativistic stars, Phys. Rev. D 102, 044048 (2020). 
[90] D. Brizuela, J. M. Martín-García, and G. A. Mena Marugan, xPert: Computer algebra for metric perturbation theory, Gen. Relativ. Gravit. 41, 2415 (2009).

[91] J. M. Martín-García, xPerm: Fast index canonicalization for tensor computer algebra, Comput. Phys. Commun. 179, 597 (2008).
[92] xAct: Efficient tensor computer algebra for the Wolfram Language, http://www.xact.es/.

[93] C. Cherubini, D. Bini, S. Capozziello, and R. Ruffini, Second order scalar invariants of the Riemann tensor: Applications to black hole space-times, Int. J. Mod. Phys. D 11, 827 (2002). 